SOCIAL RESEARCH REPORTS

ISSN: 2066-6861 (print), ISSN: 2067-5941 (electronic)

\title{
LONELINESS AND ALCOHOL ADDICTION: CASE STUDY
}

Alexandra Mihaela FERLAI

Social Research Reports, 2019, Vol. 11, Issue 3, pp. 140-152

The online version of this article can be found at:

www. researchreports.ro

https://doi.org/10.33788/srr11.3.10

Published by:

Expert Projects Publishing House

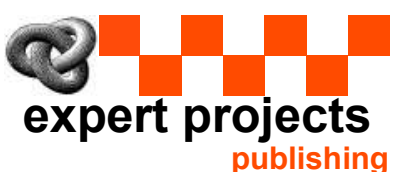

Covered by Index Copernicus International www.indexcopernicus.com

Directory of Open Access Journals www.doaj.org

On behalf of:

Center for Program and Social Development

Aditional services and information about Social Research Reports can be found at: www.researchreports.ro 


\title{
LONELINESS AND ALCOHOL ADDICTION: CASE STUDY
}

\author{
Alexandra Mihaela FERLAI ${ }^{1}$
}

\begin{abstract}
The theme of loneliness is one with a very long history, in ancient times being debated by philosophers and usually regarded from a positive point of view. The present work does not highlight the positive aspects of loneliness, but aproaches loneliness as a negative experience that accompanies the use of alcohol. Thus, we discuss about loneliness as the way in which individuals perceive, experience and evaluate the lack of communication with others. Then, we examine the relationship between loneliness and alcohol use, either as a cause, effect or maintenance factor. The paper highlights the interplay between loneliness and alcohol use and its importance in specialised treatment of the alcoholics. Also, the purpose of this paper is to illustrate the imperative need for the specialists to adress the problems that our clients deal with, from more that just a biological perspective. The case study illustrates a female's three attempts to escape alcohol dependence, each of the three attempts, enriching the intervention in order to compose a multi-faceted intervention, that we hope it will, ultimately, prove to be complete.
\end{abstract}

Keywords: loneliness, alcohol addiction, case study, social isolation, emotional loneliness

\section{Introduction}

The theme of loneliness is one with a history that goes back a long time, to Antiquity, when it was a topic debated by philosophers. They referred to positive loneliness, a concept used in German literature until 1945 and defined as the voluntary withdrawal from the daily agitation of life and oriented toward higher goals, such as reflection, meditation and communication with God. The present work does not, however, take into account the positive loneliness, but the loneliness as the way in which individuals perceive, experience and evaluate the lack of communication with others. The conceptualization and definitions of

1 Medical Center „Laura Catana”, Pianu de Jos, ROMANIA. E-mail: mihaelaferlai@ yahoo.com 
loneliness will be discussed below. Freud never spoke specifically about loneliness, but talks about the fear of being alone in his chapters on anxiety . He illustrates this with an anecdote about a child who fears the darkness unless his aunt talks to him. The child says: "If someone speaks, it becomes brighter" (p. 407).

\section{Developmental perspective}

An important factor in developing loneliness in children is the relationship with their peers. A study conducted on neglected children (neither liked nor disliked by others) and rejected children (actively disliked by others) showed that neglected but not rejected children had higher loneliness status than other children (Asher \& Wheeler, 1985; Parker \& Asher. 1987. 1993; Parkhurst \& Asher. 1992). Moreover, among those who were rejected, those who were rejected because they were submissive felt lonelier than those who were rejected because they were aggressive (Parkhurst \& Asher. 1992) and not all rejected children felt alone. The loneliness of those rejected by the group was depreciated if the rejected child maintained one friendship (Parker \& Asher, 1993). Not all children with poor interpersonal relationships (not even all neglected children) or those with poor parental attachment feel alone. One possible answer to the question which of the children at risk of loneliness actually feel alone can be found in the work of Fox et al. (Fox.1989a; Fox et al., 1994; Fox, Schmidt, Calkins, Rubin \& Coplan, 1996). They showed that children with negative affectivity who face peer rejection are more inclined to experience loneliness than children rejected by other children but who have no negative affectivity as a trait.

\section{Cognitivist approaches to loneliness}

The cognitive discrepancy theory, defines loneliness as a consequence of distorted social perceptions and attributions. Specifically, loneliness is defined as the distress that occurs when an individual's social relationships are perceived as less satisfying than what is desired (Peplau \& Perlman, 1982). From a cognitive discrepancy perspective, it is clear that loneliness is not synonymous with being alone, nor does the presence of others guarantee protection against feelings of loneliness (Peplau \& Perlman, 1982). Rather, discrepancies between ideal and perceived interpersonal relationships produce and maintain a feeling of loneliness. Loneliness is an unpleasant experience that occurs when a person's social relations network is significantly deficient, either qualitatively or quantitatively. This definition has three points of view in common with how most researchers describe loneliness. First, loneliness results from a deficiency in a person's social relations. Loneliness occurs when there is a mismatch between a person's actual social relations and the person's needs or desires for social contact. Secondly, loneliness is a subjective experience, not synonymous with objective social isolation. People can feel alone without being alone, or feel alone in a crowd. Third, the experience of loneliness is aversive. Although loneliness can be an incentive for personal development, the experience itself is unpleasant and painful. 
De Jong Gierveld takes into account the values, norms and standards of a person's life and those of in the society in which lives. From this perspective, loneliness is defined as an unpleasant or inadmissible lack in relationships or in the quality of the relationships. This includes the situation where the number of existing relationships is smaller than is considered desirable or admissible, as well as the situation where the intimacy desired by the individual is not achieved. Thus, loneliness involves the way in which a person perceives, lives and evaluates one's own isolation or lack of communication with others. This definition considers loneliness a multidimensional phenomenon, differentiating three dimensions: feelings associated with the absence of an intimate attachment relationship, feelings of inner emptiness and abandonment. The central concept is the so-called "deprivation" component. The second component refers to the temporal perspective, the way people see the future evolution of their loneliness. The third component involves different types of emotional aspects, such as pain, sadness and feelings of shame, guilt, frustration and despair.

\section{Social skills deficits}

Another conceptual approach to loneliness focuses on the deficit of social skills and personality traits, which impedes the formation and maintenance of social relationships. Research in the field of social skills has shown that loneliness is associated with increased focus on one's own person, lower skills in terms of attention to partners, a lack of self-disclosure with friends, especially among women, and reduced participation in organized groups, especially among men (reviewed in Marangoni \& Ickes, 1989). Personality research has shown that loneliness is associated with depressive symptoms, shyness, neuroticism, low selfesteem, optimism, low awareness and low levels of agreeableness (Marangoni \& Ickes, 1989). Early studies have suggested that correlates of loneliness regarding behavior and personality tend to apply only to individuals with chronic loneliness, not to those with state loneliness, circumstantial ("state-lonely" individuals) whose loneliness is adequately explained by situational factors (for example, widowhood, geographical relocation) (Marangoni \& Ickes, 1989). More recently, however, it has been observed that loneliness behaves as a personality trait even when induced in an acute manner. John Cacioppo conducted a study in 2006 in wich, under hypnosis, young adults were made to feel alone and then socially connected (or vice versa, in a counterbalanced order), recalling a time when they felt rejected and lacked the feelings of belonging, or a time when they were accepted and they felt like they belonged. Compared to their basic levels of loneliness, people made to feel lonely through hypnotic suggestions reported significantly more negative disposition and lower self-esteem, low levels of optimism, social skills, social support, sociability, extraversion, and agreeableness, and higher levels of shyness, anxiety, anger, fear of cognitive assessments and neurosis (Cacioppo, Hawkley, et $a l ., 2006)$. These results place loneliness as a potential causal factor of self-esteem, depressive symptoms, shyness, etc.

From a social needs perspective, Robert S. Weiss (1973) outlines a theory of attachment in which loneliness is determinated by deficiencies in social relationships that serve specific functions (for example, attachment, social integration, care) . 
Weiss described loneliness as "a chronic distress without redeeming features" (Weiss, 1973: 15) and distinguished between social loneliness (for example, lack of social integration), and emotional loneliness (e.g., lack of attachment persons).

\section{Evolutionary approach}

The evolutionary analysis, wich emphasizes inclusive fitness, questions the conceptualization of loneliness as an aversive condition without compensatory characteristics and instead defines loneliness as an aversive condition that promotes inclusive fitness by signaling the breakdowns of social connections. The aim of this signal is to motivate the repair or replacement of these connections (Cacioppo, Hawkley, et al., 2006). For many species, cubs need little or no parenting to survive and breed. In contrast, Homo sapiens, is born with the longest period of extreme dependence compared to any other species. Mere reproduction, therefore, is not sufficient to ensure that one's genes will reach the genetic background. In order for the genes to reach the genetic background, the cubs have to survive to breed. Moreover, the social connections and the behaviors in which they engage (cooperation, altruism, alliance) increase the survival and reproduction of those involved, increasing inclusive fitness. People have populated the Earth as hunter-gatherers for tens of thousands of years, often in conditions with numerous shortcomings. The hunter-gatherers, who would have chosen not to return to share their food and to provide protection for the mother and child (for example, who felt no loss in breaking social / family ties), would probably have managed to survive. They reproduce again, but their descendants and, with them, their genes, would have had much lower chances of surviving and reproducing. In contrast, hunter-gatherers whose genetic predisposition was inclined to share food with their family, although it would have reduced their chances of survival, would have increased their offspring's chances of survival, and thus the spread of their genes. Of course, a hunter-gatherer, who survives a hunger, can live long enough to start another family, suggesting that no single strategy is necessarily the best. Such an evolutionary scenario suggests that people may have inherited different tendencies to experience loneliness. Adoption and twin studies in children and adults have confirmed that loneliness has a considerable hereditary component (Boomsma, et al., 2005).

\section{Loneliness and alcohol use/abuse and addiction}

In 1956, Bell conducted a pioneer study of loneliness and alcohol but this pioneer work was only a collection of clinical observation. Nerviano \& Gross conducted in 1976 the first empirical study, specifically on loneliness and alcohol abuse. They started the study based on the common clinical observations that showed that many chronic alcoholics have high levels of loneliness. They saw the loneliness of alcoholics either as a part of the specific social deterioration, resulted from disffunctional use of alcohol, either as a part of the affective problems that contribute or perpetuate the alcohol consumption. Also, they pointed out that despite the serious implications that loneliness might have in the recovery process, there were few instruments to objectively measure the loneliness. 
Subsequent studies confirm this link between loneliness and alcohol consumption showing that loneliness is a factor of vulnerabilit for alcohol consumption. Thus, the first reason would be the use of alcohol as a social lubricant for the purpose of facilitating interaction (Segal, 1987, Darkes \& Goldman, 1993; Monahan \& Lannutti, 2000, Hull \& Slone, 2004 ). The second reason would be the use of alcohol as a self-medication method, in this case, the loneliness motivating the alcohol abuse through its associated psychological suffering (Carrigan \& Randall, 2003).

\section{Alcohol as a social facilitator}

Alcohol has uniquely characteristics in order to be used as a tool of socialisation, particularly in facilitating connection, because of its effects on the subjective experience of those who consume it. First, alcohol is used as a facilitator in socialization because it induces higher levels of euphoria, well-being, when consumed in a group (Darkes \& Goldman, 1993; Hull \& Slone, 2004 ). More than just that, alcohol builds the unique experiential interaction, not only because of its direct effects in alterating the emotional and cognitive state of the user, but also by offering the occasion for the drinkers to share the experince with others around. In other words, alcohol may prove especially tempting to the emotionaly lonely individuals by providing the means to achieve the same state of consciousness as another (intoxicated) person. So, drinking is "one of the means of coping with feelings of isolation, creating an illusion of friendship and togheterness" (Segal, 1987).

\section{Alcohol as self medication}

Feelings of loneliness may also predict alcohol use to the extent that those feelings stem from the pain of being with others but feeling excluded. These feelings of beeing alone, when surrounded by others, is a uniquely painful experience and loneliness correlates positively with mental health problems like depression and anxiety (Herttua et al., 2011, Richard et al. , 2017, Caccioppo et al., 2017). Individuals suffering from this psychological pain, may use alcohol as a means of numbing psychological sufference or as a means to cope with the discomfort associated with the unmet need for social connection. This hypothesis emerges from the work showing that people use alcohol as a buffer against negative experiences generally ( Mohr, Armeli, Tennen, Temple, Todd, Clark, \& Carney, 2005), and alcohol use is highly prevalent in individuals with depression and/or anxiety (Burns \& Teesson, 2002, Butler et al., 2016).

However, these are not mutually exclusive mechanisms. It is not unsual for an individ to be motivated to use alcohol in order to numb the pain of loneliness or isolation and, at the same time, to be motivated use alcohol in order to seek out connection. Either way, regardless of the way loneliness influences the use of alcohol, it is clear that there is a positive correlation between alcohol consumption and loneliness (Hornquist, 1984). Also, loneliness is a source of differential vulnerability to alcohol problems among relatively heavy drinkers due to a lack of social supports and different perceptions of social pressures. (Sadava \& Thompson, 
1986). Thus, loneliness is significant at all stages of alcoholism, as a contributing or maintaining factor in the growth of abuse or an obstacle in attempts to give up (Akerlind \& Hornquist, 1992 ).

\section{Case study}

\section{General information}

For confidentiality reasons, we will refer to the subject of the case study, as PIF. PIF is a 45 year-old woman, graduate of the Faculty of Chemistry; she has not practiced for about 4 years, currently dealing with the family business, taking care of the house and family. She is married for 20 years, with two children aged 11 and 13. She lives with her two children in a city, and her husband lives in a town, $60 \mathrm{~km}$ away, where they developed their own business. In weekends, the family reunites in one of the two places. The reason for the family's separation is the because of the greater opportunities for education for the children in a bigger city, compared to the small town where the family has a local business.

\section{First admission to the rehabilitation centre}

The client shows up for hospitalization accusing excessive fatigue, sadness most of the time, agitation, insomnia, increased appetite, panic states, anxiety in social evaluation situations, the feeling of personal loss, feelings of worthless, states that she manages through alcohol consumption, which generates feelings of guilt and shame.

\section{History of the disorder, psychological evaluation and clinical diagnosis}

The client was also admitted to a psychiatric hospital for alcohol dependence, 3 years before the first admission in our care; There she benefited from psychiatric evaluation and received psychiatric treatment for the withdrawal syndrome and for alcohol dependence and the evolution was favorable. For 2 years the consumption stopped and the subjective state was good. Subsequently, in a stressful context (significant deterioration of her father's health), alcohol consumption began again. Two months before the time of hospitalization in our center, the client was admitted to a psychiatric hospital for the increased alcohol consumption. Again, she was evaluated by a psychiatrist and received psychiatric treatment but the evolution was not favorable, the intervention being strictly focused on the ethanol dependence, only from a medical, biological point of view, so she requested admission to the our clinic.

Since December 2014 (during Christmas holiday), the client has started abusive alcohol consumption which she claims she could no longer control. She consumes alcohol in the evening, after the children fall asleep, saying that she „cannot rest and sleep unless she drinks". However, the client claims that she does not consume alcohol daily, but only in those days when she "can not resist any longer"; thus, 
since the beginning of the current problem, the client claims that she used alcohol during the holidays, then stopped. After that, the consumption started again in the context of a health problem of her father. Following this relapse she decides to seek again specialized help, and considers it would be better to include psychological care, not only medical care, so she is admitted to our center.

The psychological evaluation was carried out through 3 sessions and we used for this purpose psychological interviews, observation and standardized psychological tests : Psychiatric Screening and Diagnostic Questionnaire (PDSQ), EMAS Anxiety Multiaxial Assessment Scales.

We used the Psychiatric Screening and Diagnostic Questionnaire (PDSQ) in order to focus the initial interview and to determine the potential areas of symptoms. Responses to PDSQ items and the discussion of the items and lead scores that meet the criteria for the folowing diagnosis: Alcohol abuse / dependence (score 6 , section point 1), Social phobia (score 11, section point 5), Major depressive disorder (score 11, section point 9), Generalized anxiety disorder (score 10, section point 8 ). Consistent with the symptomatological picture suggested by the first contacts with the client and by the observations of the clients, she meets the criteria for Major Depressive Disorder, diagnosis confirmed by the subsequent interview and by the anamnesis performed by the psychiatrist of the center. PIF did not respond affirmatively to the critical items referring to the suicidal tendency present in the major depressive disorder subscale and did not declare during the subsequent interview the presence of thoughts / behaviors that suggest the existence of the suicidal tendencies as a priority.

Her father's repeated hospitalizations since December 2014, as well as his poor state of health, are major stressors, the client considering her parent entirely dependent on her, forcing her to be permanently next to him in hospital, depriving her of sleep and proper nutrition. These hings accentuated the symptoms, and the client used alcohol as self-medication in times of major stress.

PIF meets the diagnostic criteria for Social Phobia, a fact confirmed by the subsequent interview, the client declaring that no one observes what is happening to her, but for her any social contact represents a major stress, even if she manages to carry out most of the social activities, it does so with an enormous emotional and energetic cost and avoiding a series of activities, such as eating and writing in public, using public toilets, going to parties or other meetings. She was participating only in those social events from which she could only avoid with great difficulty.

The client answered "yes" to all the items of the subscale for Generalized Anxiety Disorder, confirming also in the interview the specific symptomatology.

Following the application of the Psychiatric Screening and Diagnostic Questionnaire and the follow-up interview, we observed the presence of the symptoms specific to several anxiety disorders, which is why we decided to investigate this aspect in detail, applying the Multiaxial Assessment Scales of Anxiety.

The first scale applied was EMAS-S to evaluate the client's anxiety at the time of evaluation. Based on the total score obtained by the respondent, it can be observed that the level of anxiety as a condition is well above average (EMAS-S total score $=65, \mathrm{~T}=77$ ). Analyzing the EMAS-S subscales, we found that both the level of symptoms related to the cognitive component and the level of the 
physiological emotional symptoms are well above average (EMAS-C score $=36$, $\mathrm{T}=79$; EMAS-S-EF score $=29, \mathrm{~T}=73$ ).

Next, to evaluate anxiety as a trait, we applied EMAS-T. According to the answers given on the EMAS-T scale, we find that PIF tends to feel a slightly above average level of stress in social evaluation situations (EMAS-T-ES score $=51, \mathrm{~T}=60)$. She states that she feels much above average levels of anxiety in situations thats he perceives as daily routines (EMAS-T-RZ score $=48, \mathrm{~T}=68$ ) and average levels of anxiety in physical danger situations (EMAS -T-PF, score $=51, \mathrm{~T}=49)$ and in situations of ambiguity (EMAS-T-AM, score $=43, \mathrm{~T}=51)$. As the T-levels of the EMAS-T-RZ subscale exceed 65, it is very likely that PIF will exhibit high levels of anxiety as a feature in daily routine situations.

\section{Case conceptualisation and intervention}

After discussing the results of the psychological evaluation with the clinet, we interpret the use of alcohol as a bad strategy of managing stressful situations and as a self treatment for the other comorbidities : Social phobia, Major depressive disorder, Generalized anxiety and then we build the psychotherapy goals list. So, we concluded that the negative feelings she has are numbed by the alcohol consumption, which then generated other negative feelings (guilt, shame, fear) that are numbed again by the alcohol consumption and so on, generating a spiral of negative emotions and behaviors.

The general goals for the psychotherapeutically intervention at the first admission were to (1) Improve the overall symptomatology, not only to stop alcohol abuse, (2) Develop adaptive coping strategies to replace relying on alcohol, (3) Self valuing, regardless of others opinion (4) Achieving a flexible way of thinking. In order to achieve those goals, we defined more specific objectives: (a) Overcoming all or nothing thinking, and demandingness, (b) Increase tolerance to frustration, (c) Decrease decision based on negative future-guessing, (d) Stop mind-reading, (e) Learning to evaluate in terms of behavior, not of general value, (f) Socializing without alcohol use, (g) Reasonably planning of activities.

PIF received psychiatric treatment for all the comorbidities identified and 14 CBT sessions (including those for assessment). PIF had a general good evolution; she only had a few days of withdrawal syndrome symptoms that were efficiently controlled by psychiatric medication. She begun sleeping and eating regularly, the depressive and anxiety symptoms slowly ameliorated, she learned new skills for emotional regulation and she proved to be able to master them quickly. At reassessment she registered no clinical levels of depression or anxiety and, also, no craving for alcohol. Given the good evolution, she is discharged with the following scores on PDSQ : Depression $=2$, section point 9, Generalized anxiety $=3$, section point 8 , Social phobia $=4$, section point 5 , Alcohol abuse $=0$, section point 1 . 


\section{Results}

After discharge, she goes back home, nothing changes in her environment and she continues to take the medical treatment and begins to implement the coping skills she learned during psychotherapy. She returned to the clinic for medical and psychological reassessment once/month, all of the following assessments revealing a general good evolution. After three months, the psychiatrist begun to reduce the treatment every month, until complete elimination and the results of the intervention are maintained even after the medication stop.

\section{Relapse and second admission to the clinic}

History of the current disorder. On 27 December 2018, she requests the second admission to our clinic. Her main complaints this time are: restlessness, insomnia, irritability, emotional instability, impulsivity, aggressive behavior, hostility, feelings of loneliness, emotional isolation. In July 2018 she starts drinking again, then she becomes more and more isolated, angry and hostile but the family didn't know that she has begun drinking again. On December 2018 she decided she has to seek specialized treatment again and on 27 December 2018 she is admitted for the second time to our center.

Psychologal assessment, diagnosis and case conceptualisation. The psychological assessment reveals on PDSQ high scores for Alcohol addiction syndrome (score $=6$, section point 1 ), depressive symptoms (score $=4$, section point $=9$ ), social anxiety symptoms $($ score $=3$, section point $=5$ ), generalized anxiety symptoms (score $=5$, section point $=8$ ). After the clinical interview and the in-depth discussion of the items of the screening scale, we concluded that it does not meet the criteria for any other mental disorder. PIF declarea that she continued to use the strategies learned at the previous hospitalization and that she no longer faced the emotional problems for which she used alcohol in the past. She claims that the significant problems she faced at this second admission to the center, were feelings of emotional isolation. Although she was actively involved in social interactions at school where the children studied and in their business relationships, she was feeling a lack of sense of belonging. For these reasons, we have interpreted alcohol consumption as a strategy to ameliorate these extremely distressing emotions ("When I drink, I am completely anesthetized and I do not think about big things anymore, I no longer approach existentialist themes, but I take things as they are, small. In fact, after drinking, I can't even think coherently about the difficulties of my emotional life, so no difficult thought is too much analyzed."). Thus, at this second admission to our center ( the third intervention for her), we conceptualised the case as if she was spending too much time with too much people and she had little time for meaningfull interactions. Also, the interactions were usually superficial, so she felt lonely in a crowd, most of the time. Thinking that no one will ever be able to emotionally connect with her, she started drinking again, in order numb the emotional pain.

Psyhotherapy goals, intervention and discharge. For this second CBT intervention, we set the goals of the psychotherapy with emphasize on the quality of her relationships. The establisjed goals were : (1)Stop interpreting 
others as hostile to stop social withdrawal in relevant relationships (2) Cognitive restructuring regarding social interaction (3) Filtering social interaction and reducing the superficial ones, while increasing the meaningful ones. After psychiatric evaluation, she received psychiatric treatment for withdrawal syndrome, and a light treatment for depressive symptoms. We conducted 8 CBT sessions on deepening the interactions and reducing unnecessary and emotionally demanding social interactions. The discharge assesment indicates very low depressive symptoms, no need for alcohol and an improved general well beeing. Folowing discharge, she came for psychiatric and psychologycal assesment once a month and after 3 visits, the psychiatric treatment was significantly reduced and the general wellbeing is maintained. The monthly evaluation show significant improvement in physical, psychological and social condition. In april 2019, they closed the bussiness in the other town, where the husband lived and in may 2019, the husband moved back home, the family is reunited and they started a new bussiness. The last psychological assesment (6 June 2019) shows no significant depressive symptoms, no anxiety, no alcohol use, improvement of social interactions and no feeling of loneliness.

\section{Conclusions and discussions}

The social connections and the behaviors in which people engage (cooperation, altruism, alliance) increase the survival and reproduction of those involved, increasing inclusive fitness. The aim of the sufference associated to loneliness is to signal and motivate the repair or replacement of these connections (Cacioppo, Hawkley, et al., 2006). Man is a social creature and often, the disfunctional dependence on substance is an actual substitute for the functional dependence on others (Akerlind \& Hornquist, 1992).

This case study reconstructs a major episode in PIF's life by identifying a particular set of problematic events and relationships in the context of her strive to stop the alcohol use. It can be studied or understood only in this precise context because of the difficulties to draw precise boundaries. PIF had 3 specialized interventions for its problem related to alcohol consumption. The first intervention focused strictly on the physical dependence on alcohol and on the (physical!) problems associated with it. Although the intervention was conducted only from a biological perspective, purely medical, it worked for a period of time. This is why, when she had a relapse, she asked for the same type of treatment. This time, however, she found that it was not enough in order to recover in the long run, so she seeked another kind of intervention. This second intervention was medical and psychological, combining psychiatric medication and cognitive behavioral psychotherapy, with very good results on some of the patient's problems. The second intervention, although was found efficient for some of the problems, it didn't prove to be efficient in the long run for the alcohol problem. So, the previously diagnosed emotional problems did not reappear, however, the problem related to alcohol consumption reappeared over a period of time. The third intervention, besides standard psychiatric and psychological treatment, also addressed problems 
related to psycho-social aspects. It was still a psychological approach, from a cognitive-behavioral perspective but with a focus on the social life of the patient.

The purpose of this paper is to illustrate the imperative need for the specialists to adress the problems that our clients deal, from more that just a biological perspective. The biological aspects seem to be well studied and addressed long ago. Also, in the last decades, we managed to introduce scientifically validated protocols in psychological and social intervention. However, these interventions are often so insularly conducted that we often deal only with one part of the problems our clients are confronting. The analysis and interpretation of the illustrated case is meant to lead to a better understanding of interventions on alcohol dependence and to underline the need for a holistic approach. This integrative approach supports not only the formation of a team of specialists, but also the education of the team's specialists to use its specialty and the specific techniques from such a holistic perspective.

\section{References}

Akerlind, I., \& Hornquist, J.O. (1992). Loneliness and alcohol abuse : a review of evidences of an interplay. Sot Scr Med, 34(4). 405-414.

Asher, S. R., \& Wheeler, V. A. (1985). Children's loneliness: A comparison of rejected and neglected peer status. Journal of Consulting and Clinical Psychology, 53, 500-505.

Beckman, L.J., \& Amaro, H. (1986). Personal and Social Difficulties Faced Men Entering Alcoholism Treatment. Journal of Studies on Alcohol, 47(2), 135-145.

Bloomfield, K, Stockwell, T, Gmel, G, Rehn, N. (2003) International Comparisons ofAlcohol Consumption. Alcohol Research \& Health, 27(1), 95-109.

Boomsma, I., Willemsen, G., Dolan, C.V., Hawkley, L.C., Cacioppo, J.T., (2005). Genetic and Environmental Contributions to Lonelinessin Adults: The Netherlands Twin Register Study Dorret, Behavior Genetics.

Burns, L. \& Teesson, M. (2002) Alcohol use disorders comorbid with anxiety, depression and drug use disorders. Findings from the Australian National Survey of Mental Health and Well Being. Drug Alcohol Depend, 68(3), 299-307.

Butler, T.R., Karkhanis, A.N., Jones, S.R., \& Weiner, J.L. (2016). Adolescent Social Isolation as a Model of Heightened Vulnerability to Comorbid Alcoholism and Anxiety Disorders. Alcoholism: Clinical and Experimental Research, 40(6), 12021204.

Cacioppo, J, Norris, C., Decety, J., Monteleone, G., \& Nusbaum, H. (2009). In the Eye of the Beholder: Individual Differences in Perceived Social Isolation Predict Regional Brain Activation to Social Stimuli. Journal of Cognitive Neuroscience, 21(1), 83-92.

Cacioppo, J., \& Hughes, M. (2006). Loneliness as a Specific Risk Factor for Depressive Symptoms: Cross-Sectional and Longitudinal Analyses. Psychology and Aging , 21(1), 140-151.

Cacioppo, J., Fowler, J.H., \& Christakis, N.A. (2009). Alone in the Crowd: The Structure and Spread of Loneliness in a Large Social Network. Journal of Personality and Social Psychology, in press

Cacioppo, J., Hawkley, L.C., \& Thisted, R.A. (2010). Perceived Social Isolation Makes Me Sad: Five Year Cross- Lagged Analyses of Loneliness and Depressive 
Symptomatology in the Chicago Health, Aging, and Social Relations Study. Psychol Aging, 25(2), 453-463.

Canham, S.L., Mauro, P.M., Kaufmann, C.N., Sixsmith, A. (2016). Association of alcohol use and loneliness frequency among middle-aged and older adult drinkers. J Aging Health. 28(2), 267-284.

Carrigan, M.H., \& Randall, C.L. (2003). Self-medication in social phobia: a review of the alcohol literature. Addict Behav, 28(2), 269-284.

Coplan, R. J., Rubin, K. H., Fox, N. A., Calkins, S. D., \& Steward, S. L. (1994). Being alone, playing alone, and acting alone: Distinguishing among reticence and passiveand active-solitude in young children. Child Development, 65, 129-138.

Darkes, J., \& Goldman, M. S. (1993). Expectancy challenge and drinking reduction: Experimental evidence for a mediational process. Journal of Consulting and Clinical Psychology, 61, 344-353.

de Jong, G.J. (1998) A review of loneliness: concept and definitions, determinants and consequences, Reviews in Clinical Gerontology, 8, 73-80.

Fox, N. A., Calkins, S. D., Schmidt, L. A., Rubin, K. H., \& Coplan, R. J. (1996). The role of frontal activation in the regulation and dysregulation of social behavior during the preschool years. Development and Psychopathology, 8, 89-102.

Herttua, K., Martikainen, P., Vahtera, J., \& Kivimaki, M. (2011). Living Alone and AlcoholRelated Mortality: A Population-Based Cohort Study from Finland. PloS Med, 8(9), e1001094. https://doi.org/10.1371/journal.pmed.1001094.

Hull, J. G., \& Slone, L.B. (2004). Alcohol and self-regulation. In R. F. Baumeister \& K. D. Vohs (Eds.), Handbook of self-regulation: Research, theory, and applications (pp. 66-491). New York, NY, US: The Guilford Press.

Lorant, V., Nicaise, P., Soto,V. E., d'Hoore, W. (2013) Alcohol drinking among college students: college responsibility for personal troubles. BMC Public Health, 13, 615.

Marangoni, C \& Ickes, (1989) W, Loneliness: A Theoretical Review with Implications for Measurement. Journal of Social and Personal Relationships, 6, 93.

Mohr, C.D., Armeli, S., Tennen, H., Temple, M., Todd, M., Clark, J., \& Carney, M. A. (2005). Moving Beyond the Keg Party: A Daily Process Study of College Student Drinking Motivations. Psychology of Addictive Behaviors, 19(4), 392-403.

Monahan P. L, \& Lannutti, J. (2000). Alcohol as Social Lubricant Alcohol Myopia Theory, Social Self-Esteem, and Social Interaction. Human Communication Research, 26(2), 175-202.

Nerviano, V J, \& Gross, W.F. (1976) Loneliness and locus of control for alcoholic males Validity against Murray need and Cattell trait dimensions. J Elm Psycho1 32, 479.

Parker \& Ascher (1993) Frienship and friendship quality in middle childhood: links with peer group acceptance and feelings of loneliness and social dissatisfaction. Developmental Psychology, 29(4), 611-621.

Parker, G. (1987). Becoming friends: Conversational skills for friendship formation in young children. In J. M. Gottman \& J. G. Parker (Eds.), Conversations of friends: Speculations of affective development (pp. 103-138). New York: Cambridge University Press.

Parkhurst, J.T., \& Ascher, S.R. (1992). Peer rejection in middle school : subgroup differences in behavior, loneliness and interpersonal concerns. Developmental Psychology, 28, 231-241. 
Parkhurst, J.T., \& Asher, S.R. (1987). The social concerns of aggressive-rejected children. Paper presented at the biennial meetings of the Society for Research in Child Development, Baltimore, MD.

Peplau, L. A., \& Perlman, D. (1982). Perspective on loneliness. In L. A. Peplau \& D. Perlman (Eds.), Loneliness: A sourcebook of current theory, research and therapy (pp. 1-18). New York: Wiley-Interscience

Richard. A., Rohrmann, S., Vandeleur, C.L., Schmid, M., Barth, J., \& Eichholzer, M (2017) Loneliness is adversely associated with physical and mental health and lifestyle factors: Results from a Swiss national survey. PLoS ONE, 12(7), e0181442. doi: 10.1371/journal.pone.0181442.

Rubin, K.H., \& Coplan, R.J. (2004). Paying Attention to and Not Neglecting Social Withdrawal and Social Isolation, Merrill-Palmer Quarterly, 50(4), 506-534.

Russell, D. , Peplau, L. A.. \& Ferguson, M. L. (1978). Developing a measure of loneliness. Journal of Personality Assessment, 42, 290-294.

Sadava, S. W., \& Thompson, M. M. (1986). Loneliness, social drinking, and vulnerability to alcohol problems. Canadian Journal of Behavioural Science 18(2), 133-139.

Segal, B.M. (1987). Drinking motivation and the causes of alcoholism: An overview of the problem and a multidisciplinary model. Alcohol \& Alcoholism, 22(3), 301-311.

Veenstra, M.Y., Lemmens, P. H. H. M, Friesema,I.H.M., Tan, F.E.S.,. Garretsen, H.F.L., Knottnerus, J.A. \& Zwietering, P.J. (2007). Coping style mediates impact of stress on alcohol use: a prospective population-based study. Addiction, 102, 1890-1898.

Weiss, R.S. (1973). Loneliness: The Experience of' Emotional and Social Isolation, Cambridge: MIT Press. 